\title{
Analytical and experimental issues in Ni-Mn-Ga transducers
}

\author{
LeAnn E. Faidley ${ }^{a}$, Marcelo J. Dapino ${ }^{a}$, Gregory N. Washington ${ }^{a}$, \\ Thomas A. Lograsso ${ }^{b}$, Ralph C. Smith ${ }^{c}$ \\ ${ }^{a}$ Department of Mechanical Engineering, The Ohio State University, Columbus, OH 43210 \\ ${ }^{b}$ Department of Materials Science and Engineering, Iowa State University, Ames, IA 50011 \\ ${ }^{c}$ Center for Research in Scientific Computation, North Carolina State University, Raleigh, NC 27695
}

\begin{abstract}
Ferromagnetic shape memory martensites in the Ni-Mn-Ga system have been demonstrated to achieve a number of the criteria required for next generation actuators including the production of large theoretical strains up to $6 \%$. The large strain originates in the rotation of twin variants and associated twin boundary motion which occurs in response to magnetic fields. The magnetic activation holds promise in actuator design because it can lead to higher bandwidths than those achieved through pure martensite-austenite phase transformation, as is the case with thermally-activated shape memory alloys. In this paper, we report on experimental measurements collected from a $\mathrm{Ni}_{49.0} \mathrm{Mn}_{30.0} \mathrm{Ga}_{21.0}$ cylindrical sample alloy, driven as cast by a collinear magnetic field-stress pair. Despite the lack of a known restoring force and the fact that no "training" procedures are applied, quasistatic strains as large as $4300 \mu \epsilon$ are shown. Furthermore, dynamic results in the DC-20kHz range are presented which would suggest the presence of a $\Delta \mathrm{E}$ effect similar to that seen in Terfenol-D but exhibiting an opposite dependence of stiffness with DC field. The potential implications of the results for the design and control of dynamic structures based on Ni-Mn-Ga are very significant.
\end{abstract}

Keywords: Ni-Mn-Ga, ferromagnetic shape memory alloys, $\Delta \mathrm{E}$ effect

\section{INTRODUCTION}

Large magnetic field-induced strains, up to a theoretical maximum of $6 \%$, have been reported for Ni-Mn-Ga Heusler alloys that exhibit shape memory transformations. ${ }^{1}$ These large strains occur as a result of twin boundary motion as martensite variants rotate between the direction perpendicular and parallel to the stress direction. These field-induced strains do not result from traditional Joule magnetostriction due to the alignment of magnetic moments (typically only $250 \mu \epsilon$ for these alloys) but from the reorientation of magnetically favorable martensitic variants through twin boundary motion. ${ }^{2}$ For the tetragonal martensite, the favorable variants are those whose c-axis is aligned with the field direction. Field driven twin boundary motion thus produces an overall contraction of the sample. Upon removal of the field, there is no restoring force to drive the twin boundary in the opposite direction and the field-induced strain is not recoverable.

To achieve large reversible magnetic field-induced strains, a compressive stress is applied perpendicular to the field direction that favors variants with the c-axis aligned along the compression axis. This perpendicular drive configuration is illustrated in Figure 1(a). For this paper, experiments were conducted with the stress and magnetic field applied collinearly along the magnetic easy axis direction of the sample, contrary to the conventional perpendicular arrangement (see Figure 1(a)-(b)). Despite the lack of a known restoring force, reversible strains as large as $4300 \mu \epsilon$ are demonstrated, which represent a three-fold improvement over TerfenolD. These reversible field-induced strains are seemingly too large to result from Joule magnetostriction nor can they be explained by the existing martensite variant reorientation models. The sample used for these tests is

Further author information:

L.E.F.: faidley.1@osu.edu, (614) 292-8685

M.J.D.: dapino.1@osu.edu, (614) 688-3689 (Correspondence)

G.N.W.: washington.88@osu.edu, (614) 292-8486

T.A.L.: lograsso@ameslab.gov, (515) 294-8425

R.C.S.: rsmith@eos.ncsu.edu, (919) 515-7552 


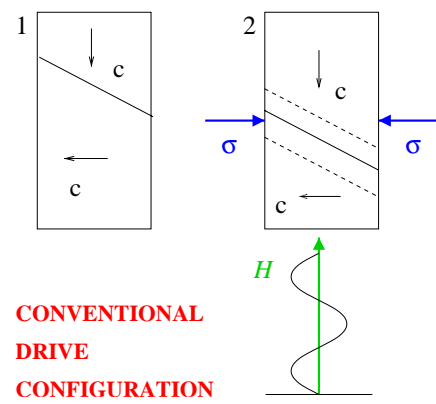

(a)
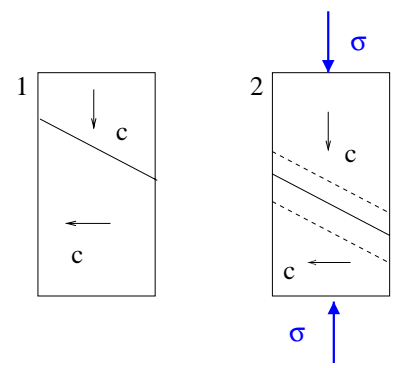

EMPLOYED

DRIVE

CONFIGURATION

(b)

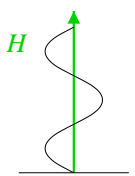

Figure 1. (a) Conventional perpendicular configuration for field-induced martensitic twin boundary motion; (b) collinear field-stress drive configuration employed in this paper.

one in a set of alloys whose compositions are centered on $\mathrm{Ni}_{49.5} \mathrm{Mn}_{29.5} \mathrm{Ga}_{21.0}$, that is lower in $\mathrm{Ga}$ and higher in Mn than the compositions believed to exhibit largest magnetic field-induced strains. This is illustrated in Fig. 2. Furthermore, contrary to the conventional processing practice of material "training" through field cycling and thermal treatments, these large strains were observed in samples tested as cast.

Despite the small changes in composition between the alloys of current interest and those showing the largest magnetic field-induced strains, significant and profound differences are apparent between the magnetic transition and shape memory transition. It has been recently reported that the room temperature structure of

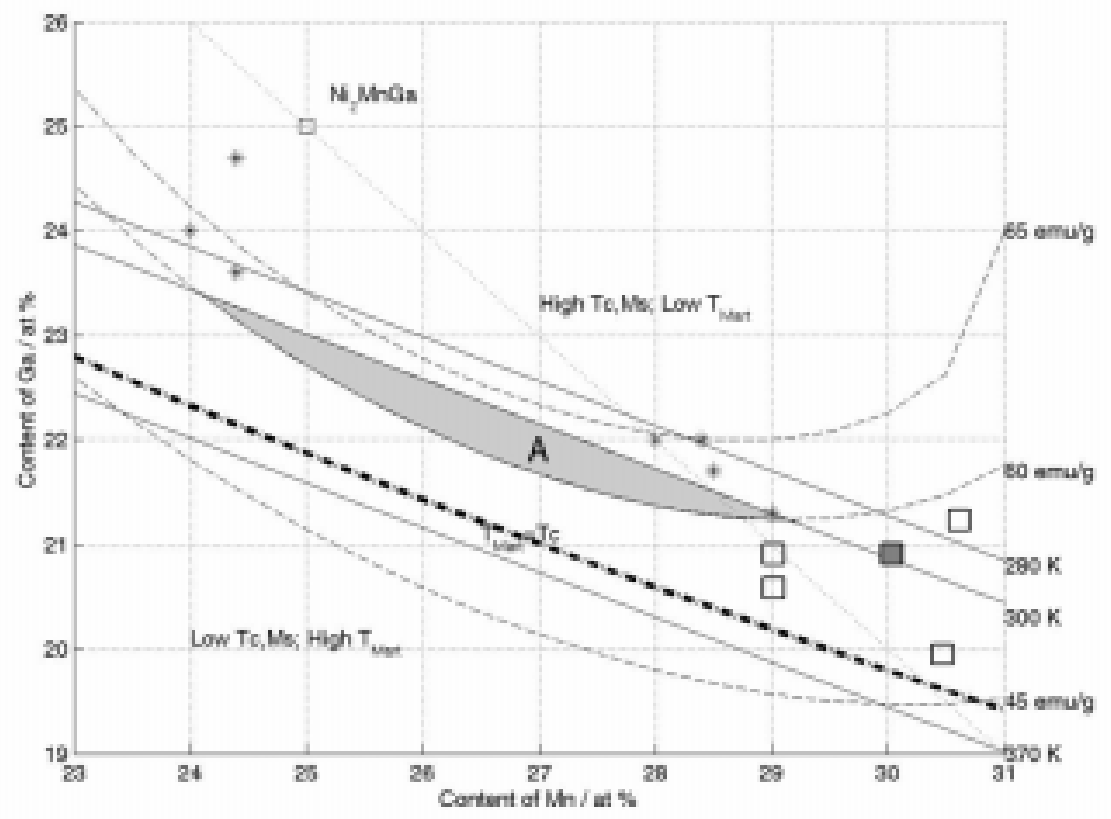

Figure 2. Compositional map for Ni-Mn-Ga alloys (taken from Jin et $\mathrm{al}^{3}$ ). The dark square represents the sample employed here. 
the martensite phase at higher Mn content is orthorhombic $(\mathrm{c} / \mathrm{a}=1.1)$ rather than tetragonal. ${ }^{4} \mathrm{~A}$ second and perhaps more significant difference is that for the sample tested, the shape memory martensitic transformation temperature are nearly coincident with the ferromagnetic ordering temperature, suggesting a coupled magnetostructural transformation which may be responsible for the observed reversible strains. Coupled transitions, known to exist in rare-earth-silicon-germanium compounds, ${ }^{5,6}$ result in extraordinary magnetic responses including large magnetostriction, where the application of a magnetic field drives the structural change from a paramagnetic austenite phase to a ferromagnetic martensitic phase. This structural change is fully reversible when the magnetic field is removed.

This paper presents experimental results collected from a $\mathrm{Ni}_{49} \mathrm{Mn}_{30} \mathrm{Ga}_{21}$ cylindrical element. A test transducer was designed and built for purposes of testing cylindrical samples with a magnetic field aligned with the [100] axis of the sample and a collinear stress loading. Data collected include transfer functions acceleration per force and acceleration per input field under various impulse, white-noise, and sine sweep excitations.

\section{EXPERIMENTS}

\subsection{Experimental Setup}

This research is focused on the dynamic testing of a single crystal alloy with composition $\mathrm{Ni}_{49} \mathrm{Mn}_{30} \mathrm{Ga}_{21}$ which was prepared by the Bridgman method. The single crystal ingot was oriented along the [100] direction and a 0.25 in $(0.635 \mathrm{~cm})$ diameter, 0.954 in $(2.423 \mathrm{~cm})$ long rod was extracted from the ingot using electrical discharge machining (EDM). The experiments were conducted with a collinear magnetic field-stress pair in the broadband research transducer shown in Figure 3, which consists of a water-cooled solenoid, pickup coil, and magnetic steel components integrated to form a closed magnetic circuit. The solenoid consists of 1350 turns of AWG 15 magnet wire and has a field rating of 167 Oe/A. Interspersed within the solenoid lies a 0.25 in-diameter copper coil which provides temperature control within $\pm 1 \mathrm{~F}$ by means of water flow at a rate of up to $6.35 \mathrm{~L} / \mathrm{min}$. The solenoid is driven by two Techron $77904 \mathrm{~kW}$ amplifiers arranged in series with an overall voltage gain of 60 and a maximum output current of $56 \mathrm{~A}$ at the nominal solenoid resistance of $3.7 \Omega$. The magnetic induction is measured with a pickup coil made from AWG 33 insulated copper wire wound in several layers around an aluminum spool and connected to a Walker Scientific MF-5D integrating fluxmeter. The strain is measured by means of a Lucas Shaevitz MHR-025 linear variable differential transducer (LVDT) while the acceleration is measured by PCB U352C22 and 352C68 accelerometers. Several Omega thermocouples are used to monitor the system temperature through a 10-channel Omega signal conditioner. For loaded tests, a fixture consisting of dead weights which can be adjusted from 0-1000lb is employed and a PCB 208C04 force transducer is used to measure stress. For impulse tests, a PCB $086 \mathrm{C} 03$ impulse hammer is used. The system is controlled by a Data Physics SignalStar Vector 550 dynamic signal analyzer and a SigLab 20-42 data acquisition system, both interfaced through a PC. The test setup is illustrated in Figure 4.

\subsection{Test Conditions}

The measured and recorded signals include either mechanical displacement or acceleration depending on whether the excitation source is quasi-static or dynamic, as well as output force, and magnetic induction. In addition, temperatures were monitored at the core of the solenoid and at the inlet and outlet of the cooling coil. Several test modalities were employed for purpose of analyzing the dynamic response of the active element in relation to the surrounding structure. Quasi-static tests were run to determine the dependence of strain and magnetization on magnetic field. In these tests, a high amplitude $(9.5 \mathrm{kOe}(756 \mathrm{kA} / \mathrm{m}) 0-\mathrm{pk})$, low frequency $(0.1,0.5,1.0$ $\mathrm{Hz}$ ) AC current was supplied to the solenoid and the strain and induction were measured using an LVDT and integrating flux-meter respectively. These tests are discussed in more detail by Malla et al. ${ }^{7}$ and are summarized in Section 4 to indicate the magnetic activity present in the sample employed in this study. The second test modality consisted of static elastic modulus characterization. In these tests, the transducer was loaded with free weights from $1.6-4 \mathrm{ksi}(11-27.5 \mathrm{MPa})$ in eleven increments and the strain was measured using an LVDT while various magnetic bias fields were applied. The results are stress vs strain curves from which the static elastic modulus was calculated as the slope of the best fit line. 


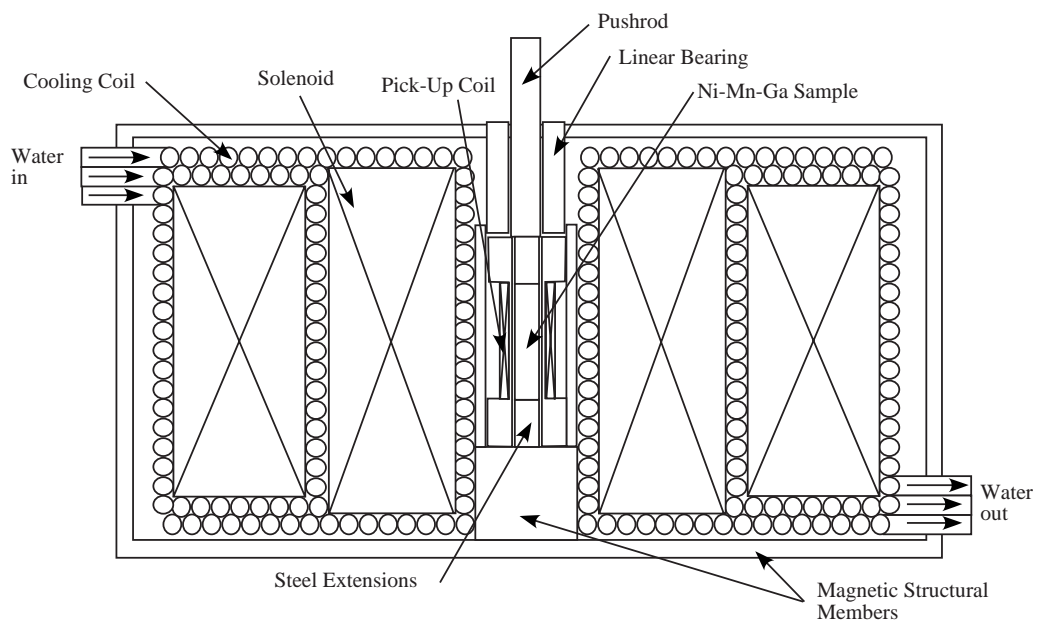

Figure 3. Cross-section of the broadband transducer employed in this study.

The third test modality and the main focus of this research was the dynamic testing of $\mathrm{Ni}_{49} \mathrm{Mn}_{30} \mathrm{Ga}_{21}$ driven by a collinear magnetic field-stress pair in the broadband transducer described in Section 2.1. The output of these tests was a series of transfer functions between the pick-up coil voltage, pushrod acceleration, and transducer housing acceleration, and the input current. The data were used to determine trends in the relationship between the stiffness (and therefore elastic modulus) of the system and the bias field. Three types of dynamic tests were performed in the range of a few Hertz to $20 \mathrm{kHz}$. First, impulse testing was employed to identify the primary mechanical resonances of the transducer-sample system. These resonances were further explored under various DC magnetic bias levels ranging from $0-1.6 \mathrm{kOe}(0-129 \mathrm{kA} / \mathrm{m})$ through the use of random excitations with an rms amplitude of $5.4 \mathrm{kOe}(431 \mathrm{kA} / \mathrm{m})$. Finally, a closer investigation of the primary resonances was achieved using swept sine analysis at these same test inputs. For these tests, the acceleration of the unloaded pushrod was measured using a PCB 352C68 accelerometer, the acceleration at the rim of the transducer was measured with a PCB U352C22 accelerometer, and the pickup coil voltage was measured directly.

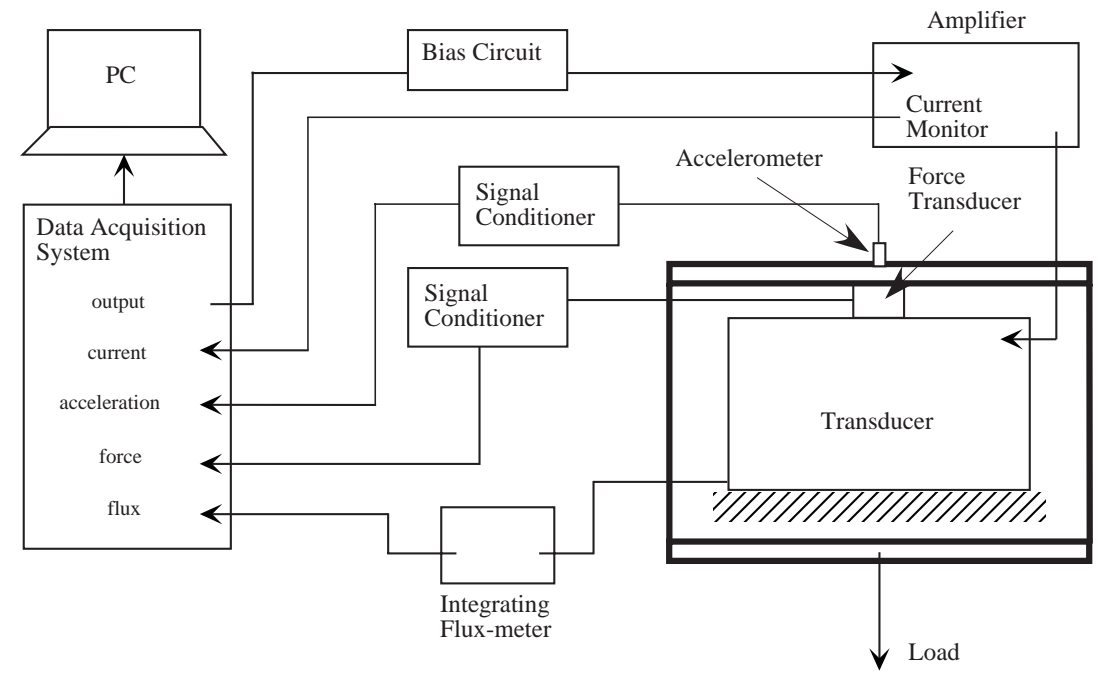

Figure 4. Experimental setup used for quasi-static and dynamic testing of the Ni-Mn-Ga samples. 


\section{MODELING AND DATA ANALYSIS}

For purposes of model development and data analysis, a two-DOF lumped-parameter model of the test transducer is considered as shown in Figure 5. Employing Newton's second law, the dynamic equations for the system take the form

$$
\begin{gathered}
m \ddot{x}=k_{1}(y-x), \\
M \ddot{y}=F-k_{1}(y-x)-k_{2} y-c \dot{y},
\end{gathered}
$$

in which zero initial conditions are assumed. Taking the Laplace transform of relations (1a) - (1b) and combining the resulting algebraic equations yields the transfer function acceleration per applied force,

$$
\frac{\ddot{x}}{F}=\frac{k_{1} s^{2}}{M m s^{4}+m c s^{3}+\left(k_{1} m+k_{2} m+M k_{1}\right) s^{2}+c k_{1} s+k_{1} k_{2}} .
$$

It is noted that the force $F$ results from the strain produced in response to the magnetic field. The mechanical resonance frequencies are determined from the poles of transfer function (2), as follows

$$
\begin{aligned}
\omega_{1}^{2} & =\frac{k_{1} m+k_{2} m+k_{1} M \pm \sqrt{k_{1}^{2} m^{2}+k_{2}^{2} m^{2}+k 1^{2} M^{2}+2 k_{1} k_{2} m^{2}+2 k_{1}^{2} M m-2 M m k_{1} k_{2}}}{2 M m}, \\
\omega_{2}^{2} & =\frac{k_{1} k_{2}}{M m \omega_{1}^{2}}
\end{aligned}
$$

where the damping $c$ between the transducer and the floor has been assumed negligible, and $s=j \omega$. Solving for $k_{1}$ and $k_{2}$ from relations $(3 \mathrm{a})-(3 \mathrm{~b})$ gives

$$
\begin{aligned}
k_{1} & =\frac{\left(\omega_{1}^{2}+\omega_{2}^{2}\right) M m \pm \sqrt{\left(\omega_{1}^{2}+\omega_{2}^{2}\right)^{2} M^{2} m^{2}-4(M+m) M m^{2} \omega_{1}^{2} \omega_{2}^{2}}}{2(M+m)}, \\
k_{2} & =\frac{M m \omega_{1}^{2} \omega_{2}^{2}}{k_{1}} .
\end{aligned}
$$

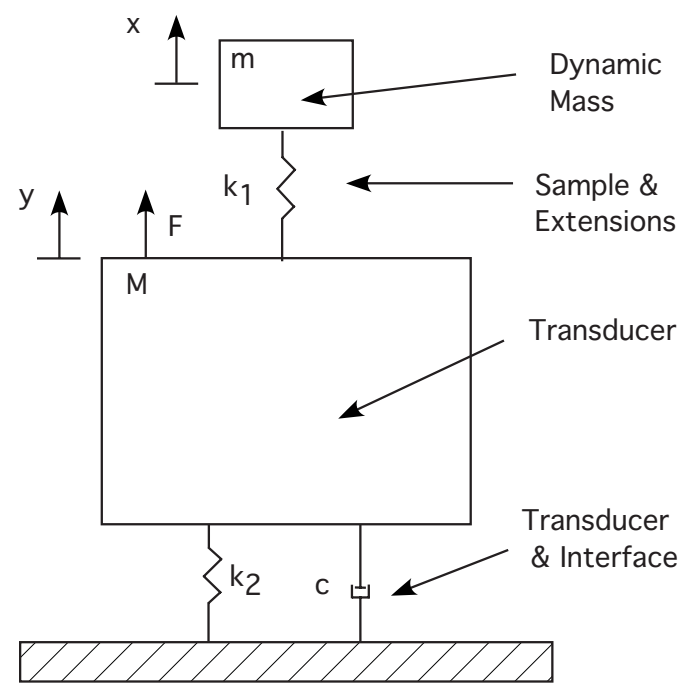

Figure 5. Two-degree of freedom lumped-parameter model of the FSMA transducer. 
Referring to Figure 5 , it is noted that $k_{1}$ represents the effective stiffness of the series combination of the pushrod stiffness $k_{p r}$, FSMA sample stiffness $k_{\text {sam }}$, and two steel extensions with stiffness $k_{\text {end }}$. Thus, the stiffness of the Ni-Mn-Ga sample takes the form

$$
k_{\text {sam }}=\frac{1}{\frac{1}{k_{1}}-\frac{1}{k_{p r}}-\frac{2}{k_{\text {end }}}} .
$$

The elastic modulus of the Ni-Mn-Ga sample $E_{\text {sam }}$ is obtained by employing a linear stiffness model $E_{\text {sam }}=$

$k_{\text {sam }} \frac{L}{A}$, where $L$ and $A$ respectively represent the length and cross-sectional area of the Ni-Mn-Ga rod. Thus, the elastic modulus takes the form

$$
E=\frac{\frac{L}{A}}{\frac{1}{k_{1}}-\frac{1}{k_{p r}}-\frac{2}{k_{\text {end }}}} .
$$

Calculations of elastic moduli under various bias fields are provided in Section 4 along with comparisons with quasi-static elastic moduli data obtained from the slope of stress-strain measurements.

\section{EXPERIMENTAL RESULTS AND DISCUSSION}

\subsection{Quasi-Static Tests}

The strain and magnetization output of various alloys centered on $\mathrm{Ni}_{49.5} \mathrm{Mn}_{29.5} \mathrm{Ga}_{21.0}$ were investigated at high amplitude, low frequency AC magnetic fields, zero magnetic bias, and various loads. While the complete set of tests are discussed in detail by Malla et al., ${ }^{7}$ we present quasi-static data for the purpose of highlighting the degree of activity of the sample used in the dynamic tests as compared to the most active alloy in the set being investigated.

Figure 6 shows strain and magnetization curves for two Ni-Mn-Ga samples at an AC magnetic field of 9.5 $\mathrm{kOe}(756 \mathrm{kA} / \mathrm{m})$ applied to an unloaded, unbiased transducer at frequencies of 0.1, 0.5, and $1 \mathrm{~Hz}$. Figures 6(a) and 6(b) show the $\mathrm{Ni}_{49.0} \mathrm{Mn}_{30.0} \mathrm{Ga}_{21.0}$ sample that was used for the dynamic tests discussed in the following sections. Figure 6(c) and (d) are data from the $\mathrm{Ni}_{48.1} \mathrm{Mn}_{30.6} \mathrm{Ga}_{21.3}$ sample that showed the largest strain of the set tested under quasi-static conditions.

In Figure 6, it is first noted that the strain increases from about 1200 to about $4300 \mu \epsilon$ as the Ni content decreases at virtually constant Ga content. This maximum represents a significant improvement over the saturation strain of Terfenol-D of about $1600 \mu \epsilon$. This increase in strain is accompanied by an increase in the magnetization hysteresis and thus results in a change in the coercivity point and permeability as is detailed in Malla et al.. ${ }^{7}$ Secondly, it is noted that there are significant changes in the transducer behavior as the excitation frequency is varied from 0.1 to $1.0 \mathrm{~Hz}$. For example, the magnetic hysteresis increases and the cross over point in the strain versus field curve also increases with increasing frequency. These observations motivate in part the investigation of the behavior under various frequencies through the use of impulse, white-noise, and swept sine excitations as discussed in later sections.

\subsection{Static Elastic Modulus Tests}

A series of static tests were conducted in order to determine the elastic modulus of $\mathrm{Ni}_{49.0} \mathrm{Mn}_{30.0} \mathrm{Ga}_{21.0}$. For these tests, the transducer was loaded at eleven increments between $1.6-4 \mathrm{ksi}(11-27.5 \mathrm{MPa})$ for bias fields of $0,100,200,500,800,1000$, and 1500 Oe $(0,7.9,15.9,39.8,63.6,79.6$, and $119.4 \mathrm{kA} / \mathrm{m})$. The applied force was measured using a force transducer and the relative deflection was measured using an LVDT. The results of these tests are shown in Figure 7. The slopes of these curves were found using the polyfit Matlab command. The elastic moduli shown in Figure 7(b) are those of the sample, extensions, and pushrod combination. However, it is estimated that these values are largely due to the stiffness of the sample itself since the Ni-Mn-Ga is much softer than the steel extensions and pushrod. It is noted that the elastic modulus varies with magnetic bias by about $11 \%$. This trend will be discussed further in the following section where the static and dynamic data will be compared. 


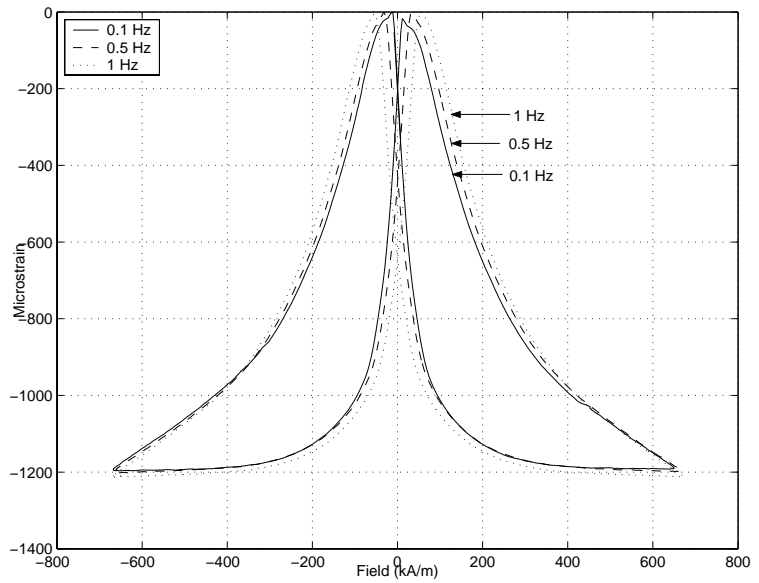

(a)

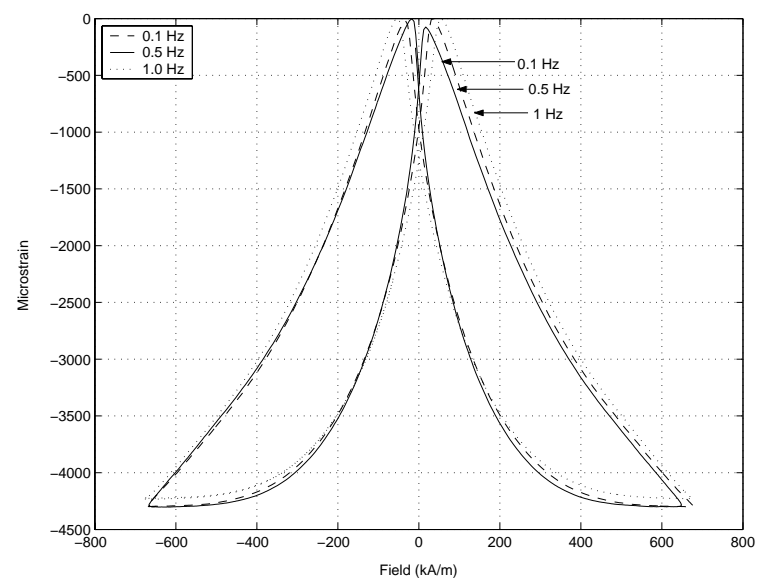

(c)

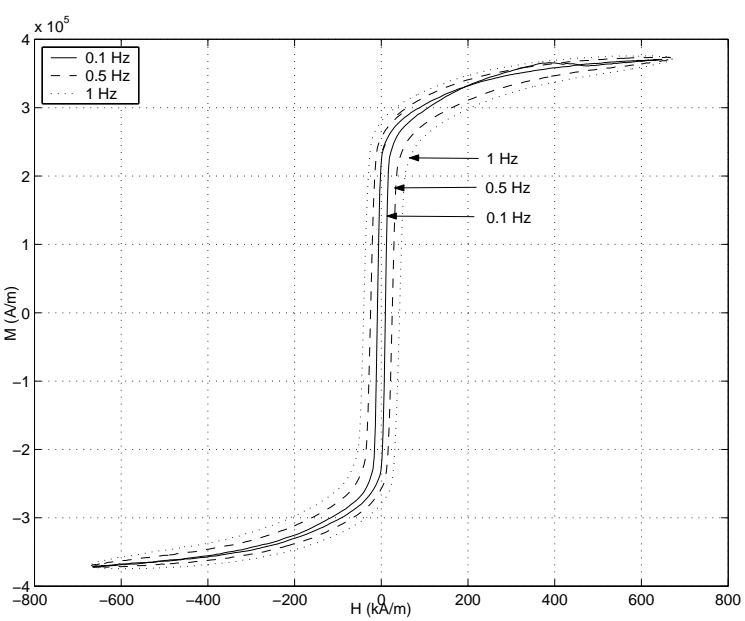

(b)

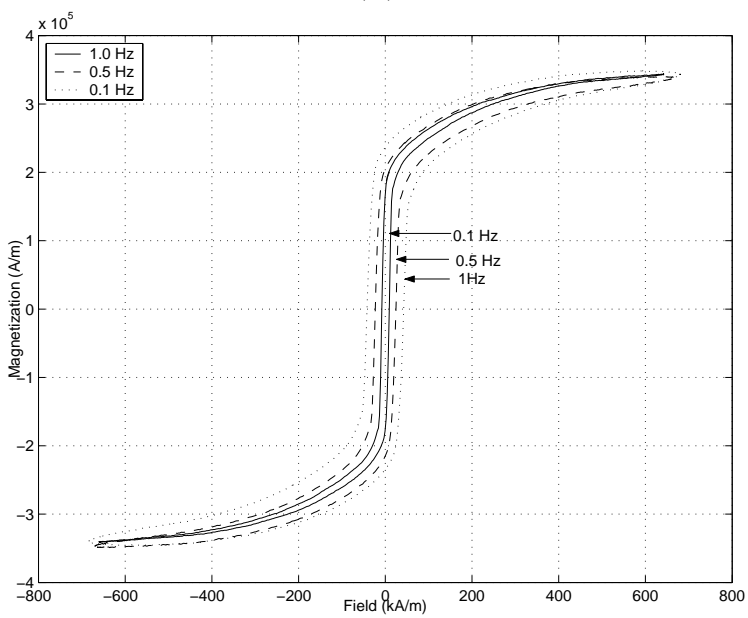

(d)

Figure 6. Quasi-static data at various low frequencies showing (a) strain vs. field and (b) magnetization vs field for $\mathrm{Ni}_{49.0} \mathrm{Mn}_{30.0} \mathrm{Ga}_{21.0}$ and (c) strain per field and (d) magnetization per field for $\mathrm{Ni}_{48.1} \mathrm{Mn}_{30.6} \mathrm{Ga}_{21.3}$.

\subsection{Frequency Response Dependence on Bias Field}

To assess the dynamic response of the transducer and Ni-Mn-Ga element, impulse, white noise, and swept sine response tests were conducted at frequencies ranging from $\mathrm{DC}-20 \mathrm{kHz}$. Figure 8 shows the transfer function pushrod acceleration per force collected for impulse excitation under no mechanical load and a shorted drive coil. The results show the approximate location of the primary system resonances at around $100-250 \mathrm{~Hz}, 1.4-1.5 \mathrm{kHz}$, and $10 \mathrm{k}-15 \mathrm{kHz}$ with the majority of resonance behavior occurring below $2 \mathrm{kHz}$.

The transducer's white-noise response is shown in Figure 9. The $1.8 \mathrm{kHz}$ peak appears to be the dominant resonance of the system and is most likely due to the vibration of the transducer casing in one of its lower modes. The $3.6 \mathrm{kHz}$ resonance is also significant and may be caused by the vibration of the Ni-Mn-Ga sample with contributions from the end extensions and pushrod.

Figure 10 shows the results from swept sine tests run at a constant AC drive field of $5 \mathrm{kOe}(400 \mathrm{kA} / \mathrm{m})$ amplitude, no mechanical load, and various DC bias fields ranging between $0-1.6 \mathrm{kOe}(0-129.3 \mathrm{kA} / \mathrm{m})$. Figures 10(a), (c), and (e) represent the transfer functions pushrod acceleration per input current for each of the aforementioned resonances while $10(\mathrm{~b}),(\mathrm{d})$, and (f) show the transfer functions canister acceleration per 


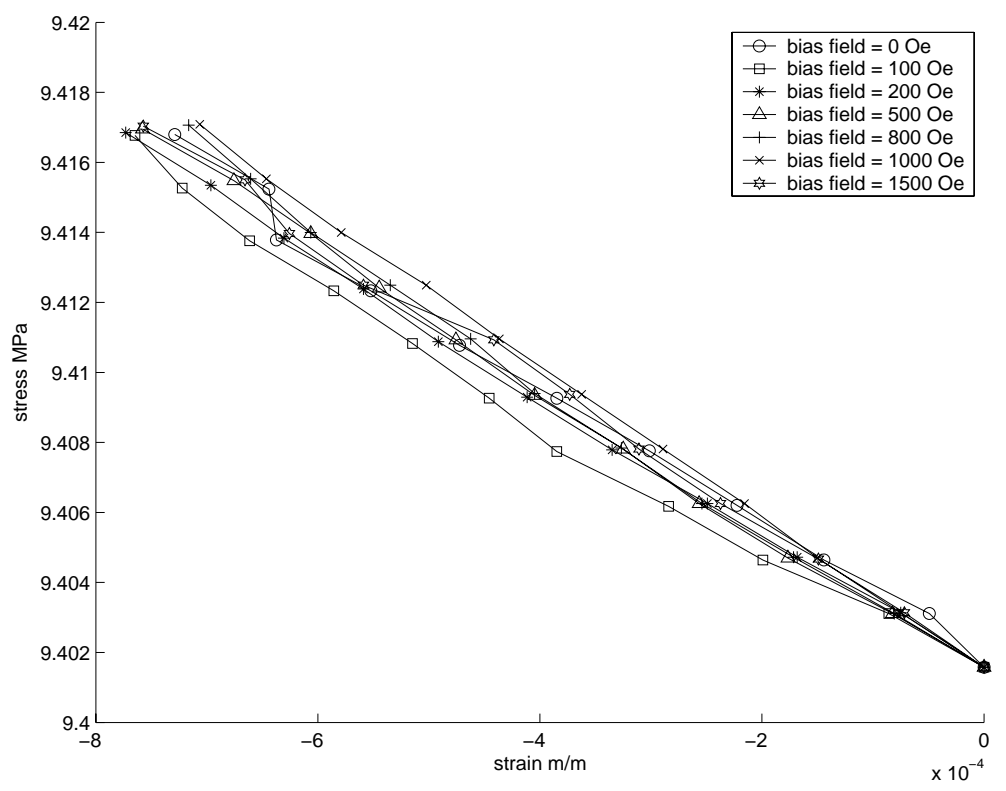

(a)

\begin{tabular}{lr}
\hline $\begin{array}{l}\text { Magnetic Bias } \\
(\mathrm{Oe})\end{array}$ & $\begin{array}{r}\text { Elastic Modulus } \\
(\mathrm{GPa})\end{array}$ \\
\hline \hline $0(0 \mathrm{kA} / \mathrm{m})$ & 19.937 \\
$100(7.9 \mathrm{kA} / \mathrm{m})$ & 19.520 \\
$200(15.9 \mathrm{kA} / \mathrm{m})$ & 19.689 \\
$500(39.8 \mathrm{kA} / \mathrm{m})$ & 20.694 \\
$800(63.6 \mathrm{kA} / \mathrm{m})$ & 21.483 \\
$1000(79.6 \mathrm{kA} / \mathrm{m})$ & 21.800 \\
$1500(119.4 \mathrm{kA} / \mathrm{m})$ & 20.297 \\
\hline Mean $\rightarrow$ & 20.489 \\
\hline
\end{tabular}

(b)

Figure 7. (a) Static stress-strain curves for various bias levels and (b) elastic modulus calculated from best fit lines.

input current. All of the plots in this figure show a dependence on magnetic bias, suggesting the presence of electromechanical coupling most likely produced by the active Ni-Mn-Ga element. In all the graphs, the amplitude of the acceleration response increases as the magnetic bias field increases. A second observed effect of increasing the magnetic bias field is the shift in the resonance frequency for most of the peaks in Figure 10. This would suggest a shift in the stiffness of the Ni-Mn-Ga sample and the associated modulus shift, known as the $\Delta \mathrm{E}$ effect. The general trend in this resonance shift seems to be a stiffening at low bias levels followed by a softening at higher levels which results in an overall softening of the sample.

It is noted that the overall modulus decrease with bias increase is the inverse of the $\Delta \mathrm{E}$ effect exhibited by magnetostrictive Terfenol-D for which an increase in DC bias field of 500 Oe $(40 \mathrm{kA} / \mathrm{m})$ produces an increase in resonant frequency of about $500 \mathrm{~Hz} .{ }^{8}$ This reversal of the $\Delta \mathrm{E}$ effect between Terfenol-D and Ni-Mn-Ga in similarly driven actuators supports the hypothesis that the physical mechanism driving the strain in these two materials is fundamentally different. 


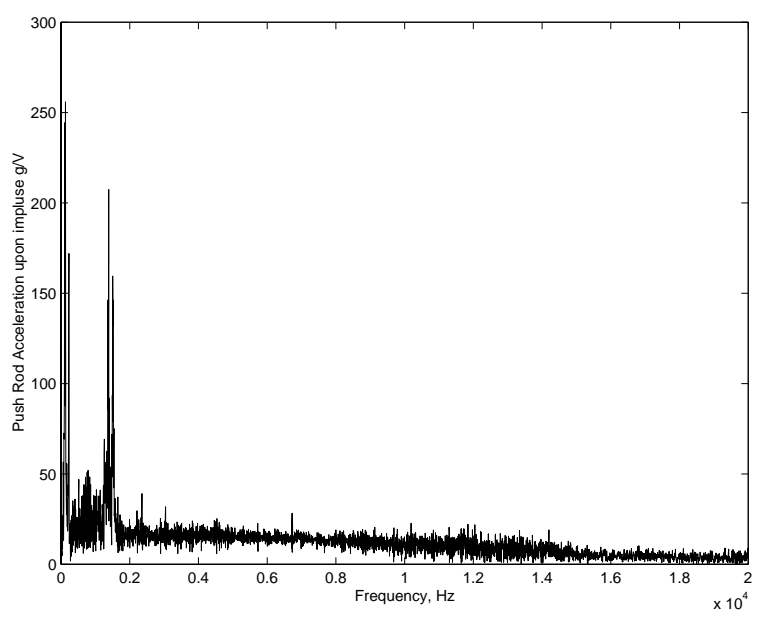

Figure 8. Transfer function pushrod acceleration per input force for impulse excitation.

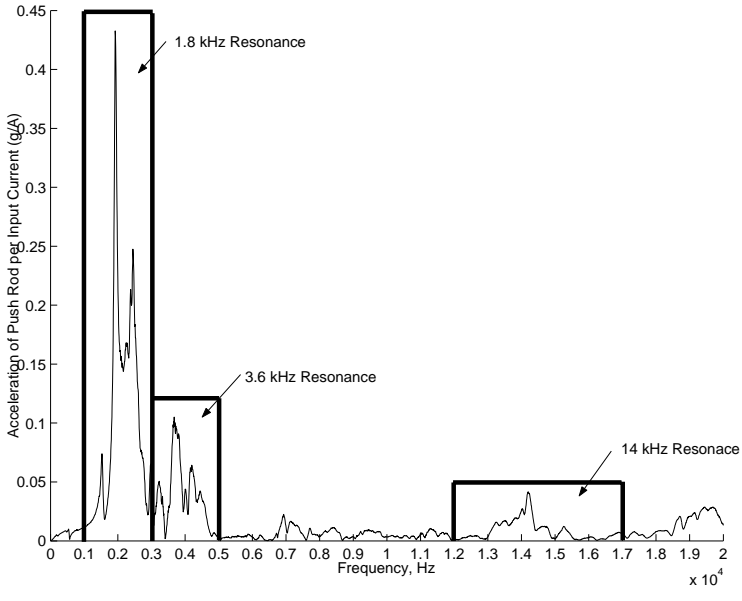

Figure 9. Transfer function pushrod acceleration per input current for white-noise excitation.

In Figure 10, it is also observed that the peak at $1.5 \mathrm{kHz}$ in (a) and at $4.5 \mathrm{kHz}$ in (d) do not show the same shift with bias field that is observed in the other peaks. These peaks are believed to be due to the vibration of components that are not coupled to the Ni-Mn-Ga sample and are therefore not affected by its stiffness change. The $1.5 \mathrm{kHz}$ peak which appears in the pushrod acceleration is uncoupled from the canister acceleration. Thus, this peak is believed to be due to a central section of the transducer that is uncoupled from the sample such as the linear bearing. The $4.5 \mathrm{kHz}$ peak seen in (d) appears only in the canister acceleration transfer function and is thus believed to represent a vibration mode from the transducer's top plate, which is structurally uncoupled from the Ni-Mn-Ga sample.

Another feature observed in Figure 10 is the fact that while the pushrod resonances and the canister resonances are aligned near the $1.8 \mathrm{kHz}$ and $14 \mathrm{kHz}$ peaks, this is not the case for the $3.6 \mathrm{kHz}$ peak. To illustrate, the frequency scale has been expanded in Figure 10(d). While the transfer function pushrod acceleration per current shows resonant response from about $3.5-4.3 \mathrm{kHz}$, the equivalent resonant response in the transfer function canister acceleration per current occurs between $3.2-3.8 \mathrm{kHz}$ while there is very little frequency content for most bias levels between $3.8-4.3 \mathrm{kHz}$. This would seem to indicate that the resonance peak seen in (c) at about $4 \mathrm{kHz}$ is very closely linked to the vibration of the Ni-Mn-Ga sample and is mostly uncoupled from the transducer. For this reason, this peak was selected as one of the two resonance frequencies employed in the analytic determination of the sample's elastic modulus.

The final step in the data analysis is the use of the equations (1)-(6) to determine the elastic modulus for the sample from the dynamic data and the comparison of these values with those determined statically. The results are shown in Figure 11 where the elastic modulus is plotted as a function of bias field for both cases. Modulus shifts of $18 \%$ and $11 \%$ are respectively calculated for the dynamic and static data. Trends show a general increase at low bias levels and decrease at higher levels for the dynamic case There are discrepancies in the turn-around point however which may be a function of a combination of the bias level spacing and testing error in the case of the static tests. The discrepancy in the magnitudes of the moduli are similar to discrepancies seen for similar tests on Terfenol- $\mathrm{D}^{9}$ and can be partially explained by the inaccuracies in the 2-DOF model used which include the lack of magnetic-structural interactions and the fact that damping was ignored in the calculations.

The high current magnitude, frequency levels, and time intervals involved in the swept sine tests seen in Figure 10 can cause test repeatability problems due to heat issues in the sample, transducer, and amplifiers. However, with adequate cooling time between tests, reasonable repeatability was achieved. This is seen in Figure 12 for a $5.4 \mathrm{kOe}(431 \mathrm{kA} / \mathrm{m}) \mathrm{AC}$ drive field and a $812 \mathrm{Oe}(64.7 \mathrm{kA} / \mathrm{m})$ bias field. 


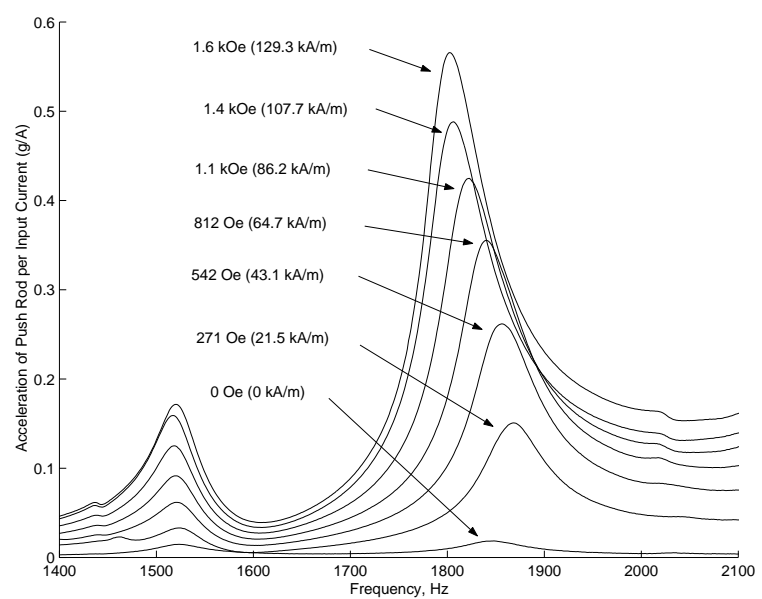

(a)

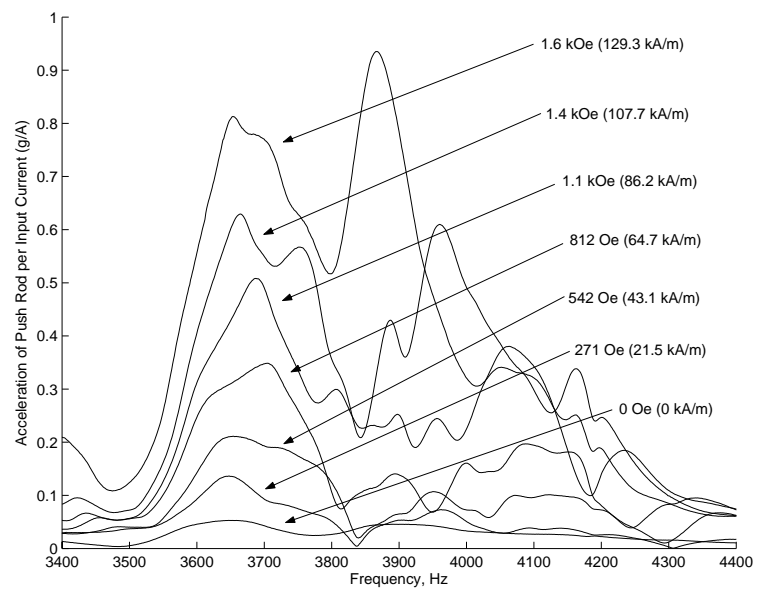

(c)

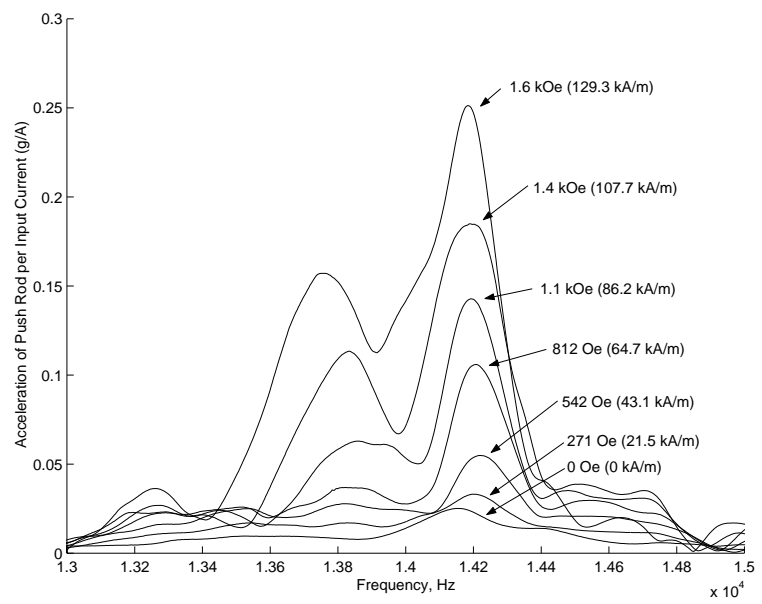

(e)

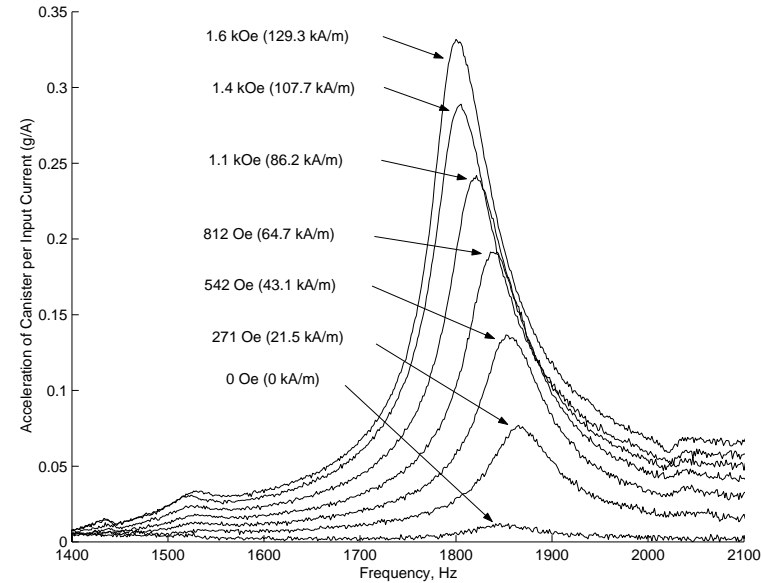

(b)

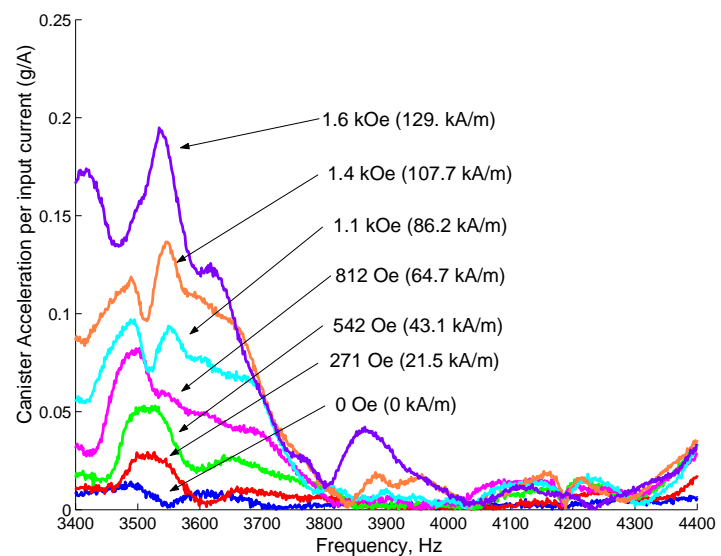

(d)

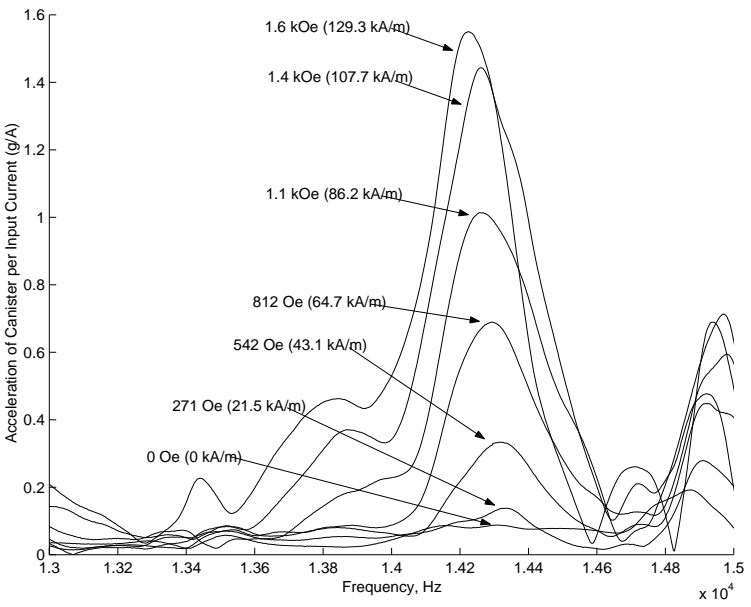

(f)

Figure 10. Swept sine response of Ni-Mn-Ga transducer at various bias fields for (a) $1.8 \mathrm{kHz}$ peak, pushrod accelerometer, (b) $1.8 \mathrm{kHz}$ peak, canister accelerometer, (c) $3.6 \mathrm{kHz}$ peak, push-rod accelerometer (d) $3.6 \mathrm{kHz}$ peak, canister accelerometer, (e) $14 \mathrm{kHz}$ peak, push-rod accelerometer, and (f) $14 \mathrm{kHz}$ peak, canister accelerometer. 


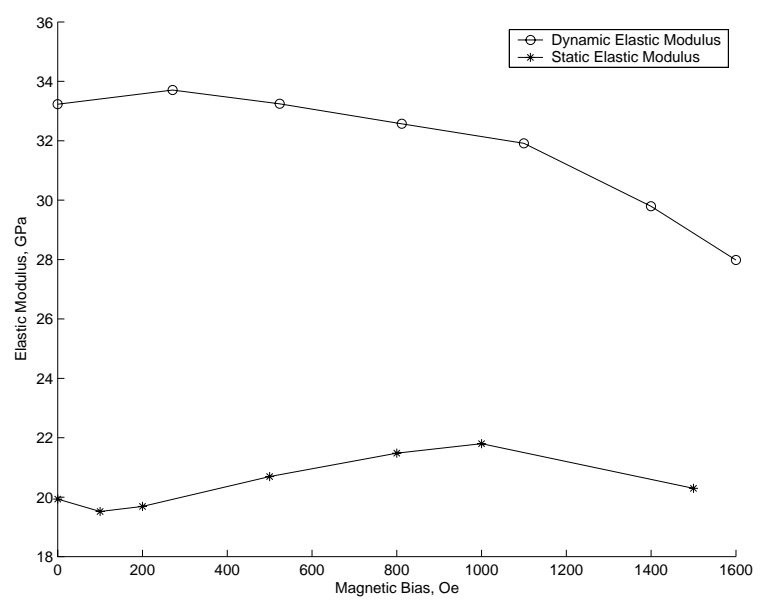

Figure 11. Comparison of elastic moduli determined from static and dynamic tests.

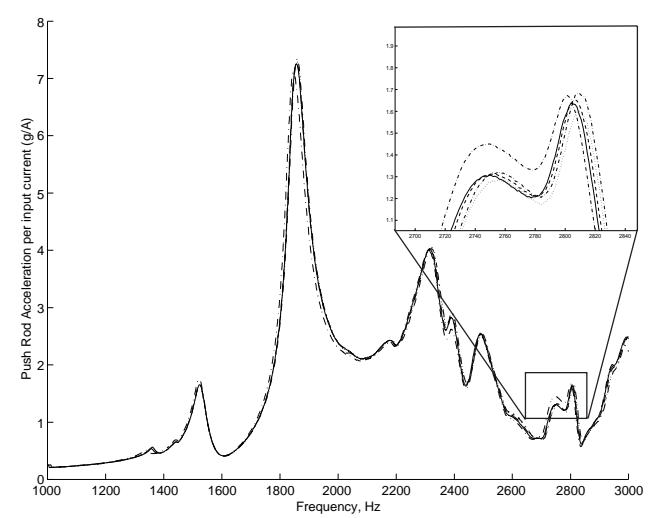

Figure 12. Repeatability data showing five identical tests. The inset shows greatest deviation.

\section{CONCLUSIONS}

This paper has presented a discussion on analytical and experimental issues involved in the use of Ni-Mn-Ga as the active element in a magnetically actuated transducer with coaxial applied field and stress. Results for tests run at frequencies ranging from DC-20 kHz with DC magnetic field levels of 0-1.6 kOe $(0-129.3 \mathrm{kA} / \mathrm{m})$ and AC field intensity of $5.4 \mathrm{kOe}(431 \mathrm{kA} / \mathrm{m})$ have shown a resonant frequency shift towards higher frequency for bias values below a certain threshold and towards lower frequencies as the bias is increased beyond that threshold. These results would suggest the presence of an $18 \% \Delta \mathrm{E}$ effect which is analogous to that seen in Terfenol-D but in which the dependence of stiffness on magnetic bias increases for low DC field values and decreases with increasing DC field after a maximum stiffness is reached. This trend is opposite to that observed in Terfenol-D. The results could enable a new class of compact ferromagnetic shape memory actuators driven with solenoids as opposed to electromagnets as is the case with current devices. Future work in this area will include the modification of the system model to include damping and mechanical-magnetic interactions as well as further testing in smaller bias increments. In addition, the effect of drive field amplitude and mechanical load will be further investigated.

\section{ACKNOWLEDGMENTS}

Financial support for L.E.F. comes from the Ohio Space Grant Consortium and The Ohio State University Graduate Fellowship program. Funding for M.J.D. was provided in part by The Ohio State University through startup funds. The research of R.C.S. was supported in part by NSF, grant CMS-0099764, and by the Air Force Office of Scientific Research, grant AFOSR-F49620-01-1-0107. T.A.L. acknowledges the support of the Office of Basic Energy Sciences, Materials Science Division, of the U.S. Department of Energy under Contract No. W-7405-ENG-82. Deborah L. Schlagel aided in the manufacturing of the sample and Rick Kellogg was influential in the development of the testing setup.

\section{REFERENCES}

1. R. O'Handley, S. Murray, M. Marioni, H. Nembach, and S. Allen, "Phenomenology of giant magnetic-field induced strain in ferromagnetic shape-memory materials," J. Appl. Phys. 87, pp. 4712-4717, May 2000.

2. H. Chopra, C. Ji, and V. Kokorin, "Magnetic-field-induced twin boundary motion in magnetic shape-memory alloys," Phys. Rev. B. 61, pp. 4913-4915, June 2000.

3. X. Jin, M. Marioni, D. Bono, S. Allen, R. O'Handley, and T. Hsu, "Empirical mapping of Ni-Mn-Ga properties with composition and valence elctron concentration," Journal of Applied Physics 91, May 2002. 
4. A. Sozinov, A. A. Likhachev, N. Lanska, and K. Ullakko, "Giant magnetic-field induced strain in nimnga seven-layered martensitic phase," Appl. Phys. Let. 80, pp. 1746-1749, March 2002.

5. V. Pecharsky and J. K.A. Gshneidner, "Giant magnetocaloric effect in $G_{5}\left(S i_{2} G e_{2}\right)$," Physical Review Letters 78, pp. 4494-4497, June 1997.

6. V. Pecharsky and J. K.A. Gshneidner, "Tunable magnetic regenerator alloys with a giant magnetocaloric effect for magnetic refrigeration from 20 to 290 k," Applied Physics Letters 70, pp. 3299-3301, June 1997.

7. A. Malla, M. Dapino, and T. Lograsso, "Effect of composition on the magnetic and elastic properties of shape memory NiMnGa," Proceedings of SPIE Smart Structures and Mateials Conf., March 2003.

8. A. Flatau, M. Dapino, and F. Calkins, "High bandwidth tunability in a smart vibration absorber," J. Intell. Mater. Syst. and Struct. 11, pp. 923-929, Dec 2000.

9. M. Dapino, F. Calkins, and A. Flatau, "On indentification and analysis of fundamental issues in Terfenol-D transducer modeling," Proceedings of SPIE Smart Structures and Materials Conf. 3392(23), 1998. 